TRANSACTIONS OF THE

AMERICAN MATHEMATICAL SOCIETY

Volume 348, Number 3, March 1996

\title{
R-TORSION AND ZETA FUNCTIONS FOR ANALYTIC ANOSOV FLOWS ON 3-MANIFOLDS
}

\author{
HÉCTOR SÁNCHEZ-MORGADO
}

\begin{abstract}
We improve previous results relating R-torsion, for an acyclic representation of the fundamental group, with a special value of the torsion zeta function of an analytic Anosov flow on a 3-manifold. By using the new techniques of Rugh and Fried we get rid of the unpleasent assumptions about the regularity of the invariant foliations.
\end{abstract}

\section{INTRODUCTION}

Let $\phi$ be a transitive analytic Anosov flow on a closed 3-manifold $M$. A representation $\rho: \pi_{1}(M) \rightarrow U(n)$ defines a flat vector bundle $E_{\rho}$. The torsion function is defined as the Euler product over the prime periodic orbits $\gamma$ of period $l(\gamma)$

$$
R_{\phi, \rho}(z)=\prod_{\gamma} \operatorname{det}\left(I-\Delta(\gamma) e^{-z l(\gamma)} \rho(\gamma)\right)^{-1}
$$

where $\Delta(\gamma)= \pm 1$ according to whether or not $E^{u}(\gamma)$ is orientable; in other words $\Delta$ is the holonomy of the orientation bundle $\left|E^{u}\right|$ of the unstable bundle. The product converges for $\Re z>>0$ and extending ideas of Rugh [7], [8], Fried [3] has constructed a meromorphic continuation to the whole complex plane.

Consider now a cell decomposition of $M$ and the corresponding cell decomposition of the universal covering $\tilde{M}$. Let $C^{i}$ be the set of equivariant $\mathbb{C}^{n}$ valued i-cochains of $\tilde{M}$. Since equivariance is preserved by the coboundary map we obtain a cochain complex

$$
C^{0} \stackrel{d}{\longrightarrow} C^{1} \stackrel{d}{\longrightarrow} C^{2} \stackrel{d}{\longrightarrow} C^{3} .
$$

If its cohomology $H^{*}\left(M, E_{\rho}\right)$ vanishes, the representation is called acyclic. In such a case there is a chain retraction $D: C^{i+1} \rightarrow C^{i}$, then an isomorphism $d+D$ : $C^{0} \oplus C^{2} \rightarrow C^{1} \oplus C^{3}$ and using a geometrical basis one defines $R$-torsion as

$$
\tau_{\rho}(M)=|\operatorname{det}(d+D)| .
$$

Extending previous results [1], [9], we prove in this paper

Theorem 1. Let $\phi$ be an analytic transitive Anosov flow on an orientable closed 3-manifold $M$. Let $\rho: \pi_{1}(M) \rightarrow U(n)$ be an acyclic representation. Suppose there is

Received by the editors November 18, 1994.

1991 Mathematics Subject Classification. Primary 58F15, 58F20, 58G10.

Key words and phrases. R-torsion, zeta functions, transitive Anosov flows.

Partially supported by DGAPA-UNAM IN-103792. 
a periodic orbit $\gamma_{0}$ such that $1, \Delta\left(\gamma_{0}\right)$ are not eigenvalues of $\rho\left(\gamma_{0}\right)$. Then the torsion function $R_{\phi, \rho}$ is regular at the origin and

$$
\tau_{\rho}(M)=\left|R_{\phi, \rho}(0)\right| .
$$

The typical example where this result applies is the unit tangent bundle $M=U S$ of an orientable closed surface $S$ with a $C^{\omega}$ Riemannian metric of negative curvature and $\phi$ the geodesic flow on $M$.

Most of the work for this paper was done during a stay at the IHES. We thank the Institute for its hospitality. We would also like to thank D. Fried and H. Rugh for helpful conversations.

\section{R-TORSION}

Along this paper $\phi$ will be a transitive analytic Anosov flow on an orientable closed 3-manifold $M$ so that the tangent bundle has a continuous splitting $T M=$ $E^{u} \oplus E^{s} \oplus E^{c}$ where $E^{u}, E^{s}$ are flow invariant line bundles exponentially contracted by $D \phi_{-t}$ and $D \phi_{t}$ respectively as $t \rightarrow \infty$ and $E^{c}$ is the line bundle generated by the vector field defining the flow.

Given a periodic orbit $\gamma$ and $x \in \gamma$, we consider the derivative $P_{\gamma}$ of the Poincaré map defined on $E_{x}^{s} \oplus E_{x}^{u}$, which can be represented in matrix form as $\left(\begin{array}{cc}S_{\gamma} & 0 \\ 0 & U_{\gamma}\end{array}\right)$. Defining

$$
\Phi_{s}(x)=\lim _{t \rightarrow 0} \frac{1}{t} \log \left|D \phi_{t}\right| E_{x}^{s} \mid \quad \text { and } \quad \Phi_{u}(x)=\lim _{t \rightarrow 0} \frac{1}{t} \log \left|D \phi_{t}\right| E_{x}^{u} \mid,
$$

we have

$$
\log \left|S_{\gamma}\right|=\int_{0}^{l(\gamma)} \Phi_{s}\left(\phi_{t}(x)\right) d t \quad \text { and } \quad \log \left|U_{\gamma}\right|=\int_{0}^{l(\gamma)} \Phi_{u}\left(\phi_{t}(x)\right) d t .
$$

We now introduce symbolic dynamics. According to Ratner [5] given $\eta>0$ there is a Markov family of size $\eta$, i.e. a finite collection of compact sets $\left\{R_{k}\right\}_{k \in I}$ contained in a family of analytic transverse discs $\left\{D_{j}\right\}$ with $\operatorname{diam}\left(R_{k}\right)<\eta$ such that

1) $\mathcal{R}=\cup_{k \in I} R_{k \in I}$ meets every flow line in time $<\eta$.

2) There is a transition set $A \subset I \times I$ such that for each $(k, l) \in A$ there is a map $f_{k l}: R_{k l} \subset R_{k} \rightarrow R_{l}$ given by $f_{k l}(x)=\phi_{t_{k l}(x)}(x)$ for $x \in \operatorname{int} R_{k l}$, where $\left.t_{k l}: \operatorname{int} R_{k l} \rightarrow\right] 0, \eta[$ is the first return time to $\mathcal{R}$ that extends to a continuous function on $R_{k l}$. The families $\left\{\operatorname{int} R_{k l}\right\}$ and $\left\{\operatorname{int} f\left(R_{k l}\right)\right\}$ are both disjoint and

$$
R_{k}=\bigcup_{l:(k, l) \in A} R_{k l}, \quad R_{l}=\bigcup_{k:(k, l) \in A} f_{k l}\left(R_{k l}\right) .
$$

Let $B_{k l}=\left\{\phi_{t}(x): 0 \leq t<t_{k l}(x), x \in R_{k l}\right\}$ and $B_{k}=\bigcup_{l:(k, l) \in A} B_{k l}$. Then $M=\bigcup_{k \in I} B_{k}$.

Let $\gamma_{0}$ be an orbit as in Theorem 1. Ratner constructed Markov families $\left\{R_{k l}\right\}_{k \in I}$ of small size such that for any $k \in I, \partial R_{k} \subset W^{s}\left(\gamma_{0}\right) \cup W^{u}\left(\gamma_{0}\right)$. We will always use such Markov families $\left\{R_{k}\right\}$ of sufficiently small size to have each $B_{k}$ simply connected. Fix a point $q \in R_{J}$ and for each $k \in I, k \neq J$ choose a point $p_{k} \in R_{k}$. For $(k, l) \in A$ choose a segment $\alpha_{k l}$ in $B_{k l}$ joining $p_{k}$ and $p_{l}$, then the union of these segments forms a direct graph which is connected since the flow is transitive. A maximal tree based at $q$ gives a directed path $\alpha_{k}$ from $q$ to $p_{k}$. For $(k, l) \in A$ let $\beta_{k l}$ be the class of $\alpha_{l}^{-1} \alpha_{k l} \alpha_{k}$ in $\pi_{1}(M, q)$. Let $\rho: \pi_{1}(M) \rightarrow U(n)$ be an acyclic 
representation, $\Delta: \pi_{1}(M) \rightarrow U(1)$ be the holonomy of the orientation bundle of the unstable bundle. Put $\rho_{k l}=\rho\left(\beta_{k l}\right), \Delta_{k l}=\Delta\left(\beta_{k l}\right)$. Let

$$
A=W^{s}\left(\gamma_{0}\right) \cap \bigcup_{k \in I} \partial B_{k} .
$$

For $\epsilon>0$ sufficiently small the interval bundle

$$
W(\epsilon):=\bigcup_{x \in A} W^{s}(x ; \epsilon)
$$

has no self-intersections and then it is a tubular neighborhood of $\gamma_{0}$. Hence, the manifold with piecewise smooth boundary $M(\epsilon):=M \backslash W(\epsilon)$ can be taken as the exterior of $\gamma_{0}$. Since 1 is not an eigenvalue of $\rho\left(\gamma_{0}\right)$, the excision property of R-torsion [2] implies that $E_{\rho} \mid(M, M(\epsilon))$ is acyclic with R-torsion given by

$$
\tau_{\rho}(M, M(\epsilon))=\tau_{\rho}(\overline{W(\epsilon)}, \partial \overline{W(\epsilon)})=\left|\operatorname{det}\left(I-\rho\left(\gamma_{0}\right)\right)\right| .
$$

Since $\rho$ is acyclic, so is $E_{\rho} \mid M(\epsilon)$ and we have

$$
\tau_{\rho}(M)=\left|\operatorname{det}\left(I-\rho\left(\gamma_{0}\right)\right)\right| \tau_{\rho}(M(\epsilon)) .
$$

Collapsing $M(\epsilon)$ along the leaves of the stable foliation we get a 2-dimensional complex $\Omega$ simply homotopic to $M(\epsilon)$ and therefore $E_{\rho} \mid \Omega$ is acyclic with

$$
\tau_{\rho}(M(\epsilon))=\tau_{\rho}(\Omega)
$$

Each $B_{k} \cap M(\epsilon)$ collapses to a 2-cell $T_{k}$, the rectangles $R_{k} \cap M(\epsilon)$ collapse to unstable intervals $U_{k}$, and the intersection of $M(\epsilon)$ with the union of the stable walls of the $B_{k}$ 's collapses to a directed graph $\Gamma \subset \Omega$ with set of vertices $J=$ boundary points of the $U_{k}$ 's. For $p \in J$, denote by $L_{p}$ the segment of $\Gamma$ from $p$ to the next point $g(p) \in J$ in the direction of the flow. Thus, periodic orbits of $g$ are in $1-1$ correspondence with the loops in $\Gamma$. We have the chain complex

$$
0 \rightarrow \bigoplus_{k \in I} \mathbb{Z} T_{k} \stackrel{\partial_{1}}{\longrightarrow} \bigoplus_{k \in I} \mathbb{Z} U_{k} \oplus \bigoplus_{p \in J} \mathbb{Z} L_{p} \stackrel{\partial_{0}}{\longrightarrow} \bigoplus_{p \in J} \mathbb{Z} p \rightarrow 0
$$

with

$$
\begin{gathered}
\partial_{1}\left(T_{k}\right)=\left(\sum_{l:(k, l) \in A} \Delta_{k l} U_{l}-U_{k}, h\left(T_{k}\right)\right), \\
\partial_{0}\left(L_{p}\right)=g(p)-p .
\end{gathered}
$$

Putting $V=\mathbb{C}^{n}$, the corresponding twisted cochain complex is given by

$$
\begin{gathered}
V^{J} \stackrel{d_{0}}{\longrightarrow} V^{I} \oplus V^{J} \stackrel{d_{1}}{\longrightarrow} V^{I} \\
d_{0}=\left(\begin{array}{c}
b \\
G-I
\end{array}\right), \quad d_{1}=\left(H-I, h^{*}\right)
\end{gathered}
$$

$b, h^{*}$ are maps we don't need to know explicitly, $(G x)_{p}=\rho\left(L_{p}\right)^{t} x_{g(p)}$ and

$$
(H y)_{k}=\sum_{l:(k, l) \in A} \Delta_{k l} \rho_{k l}^{t} y_{l} .
$$

In [9] we proved the following proposition.

Proposition 1. $G-I$ and $H-I$ are nonsingular and

$$
\tau_{\rho}(M)=\frac{\left|\operatorname{det}\left(I-\rho\left(\gamma_{0}\right)\right) \operatorname{det}(I-G)\right|}{|\operatorname{det}(I-H)|}=\frac{\left|\operatorname{det}\left(I-\rho\left(\gamma_{0}\right)\right)\right|^{2}\left|\operatorname{det}\left(I-\Delta\left(\gamma_{0}\right) \rho\left(\gamma_{0}\right)\right)\right|}{|\operatorname{det}(I-H)|} .
$$




\section{ZETA FUNCTIONS}

As in section 2, let $\left\{R_{k}\right\}$ be a Markov family with transition set $A$. Consider the usual subshift of finite type $\left(\Sigma_{A}, \sigma\right)$ and the natural map $\Pi_{A}: \Sigma_{A} \rightarrow \mathcal{R}$ such that $\Pi_{A}(\mathbf{k})$ is the point whose trajectory runs through $\cdots R_{k_{-1}} ; R_{k_{0}}, R_{k_{1}} \cdots$. Defining $\left.\tau: \Sigma_{A} \rightarrow\right] 0, \infty\left[\right.$ by $\tau(\mathbf{k})=t_{k_{0} k_{1}}\left(\Pi_{A}(\mathbf{k})\right)$, one constructs the suspension flow $(\Sigma, \psi)$ with first return time $\tau$. $\Pi_{A}$ determines a semiconjugacy $\Pi$ between $\psi$ and $\phi$ mapping periodic orbits of $\psi$ to periodic orbits of $\phi$. For $\sigma^{m}(\mathbf{k})=\mathbf{k}$ let

$$
\begin{gathered}
\rho^{m}(\mathbf{k})=\rho_{k_{m-1} k_{m}} \cdots \rho_{k_{0} k_{1}}, \quad \tau^{m}(\mathbf{k})=\tau(\mathbf{k})+\cdots+\tau\left(\sigma^{m-1} \mathbf{k}\right), \\
\Delta^{m}(\mathbf{k})=\Delta_{k_{0} k_{1}} \cdots \Delta_{k_{m-1} k_{m}} \\
D f_{\mathbf{k}}^{m}=D f_{k_{m-1} k_{0}} \cdots D f_{k_{0} k_{1}}\left(\Pi_{A}(\mathbf{k})\right)
\end{gathered}
$$

For the suspension flow we have the torsion function

$$
R_{\psi, \rho}(z)=\exp \sum_{m=1}^{\infty} \frac{1}{m} \sum_{\sigma^{m} \mathbf{k}=\mathbf{k}} \exp \left(-z \tau^{m}(\mathbf{k})\right) \Delta^{m}(\mathbf{k}) \operatorname{tr} \rho^{m}(\mathbf{k}) .
$$

There is an overcounting of periodic orbits in the symbolic flow and to arrange for this one uses the auxiliary subshifts of Manning and Bowen. For our Markov families we only need to introduce a finite number of extra factors all coming from the original orbit $\gamma_{0}$. As we saw in [9] one can write

$$
R_{\phi, \rho}(z)=R_{\psi, \rho}(z) \operatorname{det}\left(I-e^{-z l\left(\gamma_{0}\right)} \Delta\left(\gamma_{0}\right) \rho\left(\gamma_{0}\right)\right) \operatorname{det}\left(I-e^{-z l\left(\gamma_{0}\right)} \rho\left(\gamma_{0}\right)\right)^{2} .
$$

For future reference we introduce the twisted Rugh zeta function

$$
d_{\rho}(s)=\exp -\sum_{m=1}^{\infty} \frac{s^{m}}{m} \sum_{\sigma^{m} \mathbf{k}=\mathbf{k}} \frac{\operatorname{tr} \rho^{m}(\mathbf{k})}{\left|\operatorname{det}\left(I-D f_{\mathbf{k}}^{m}\right)\right|} .
$$

Recall that if $g: \Sigma_{A} \rightarrow \mathbb{R}$ is a Hölder function we define the zeta function

$$
\zeta(s, g)=\exp \sum_{m=1}^{\infty} \frac{s^{m}}{m} \sum_{\sigma^{m} \mathbf{k}=\mathbf{k}} \exp \left(g^{m}(\mathbf{k})\right)
$$

where $g^{m}(\mathbf{k})=g(\mathbf{k})+\cdots+g\left(\sigma^{m-1} \mathbf{k}\right)$. We can choose [4] a Hölder function $G$ : $\Sigma_{A} \rightarrow \mathbb{R}$ cohomologous to $g$ and depending only on future coordinates. Since $g^{m}(\mathbf{k})=G^{m}(\mathbf{k})$ whenever $\sigma^{m} \mathbf{k}=\mathbf{k}$ we have that $\zeta(s, g)=\zeta(s, G)$ and so we can think that $g$ depends only on future coordinates. The radius of convergence of $\log \zeta(s, g)$ is $\exp (-P(g))$ where $P(g)$ is the topological pressure of $g$.

For $\mathbf{k} \in \Sigma_{A}$ and $i=u, s$ let

$$
\lambda_{i}(\mathbf{k})=\int_{0}^{\tau(\mathbf{k})} \Phi_{i} \circ \phi_{t} \circ \Pi_{A}(\mathbf{k}) d t
$$

then $\lambda_{u}, \lambda_{s}$ are Hölder functions, and for $\sigma^{m}(\mathbf{k})=\mathbf{k}$ we have

$$
\operatorname{det}\left(I-D f_{\mathbf{k}}^{m}\right)=\left(1-\lambda_{u}^{m}(\mathbf{k})\right)\left(1-\lambda_{u}^{m}(\mathbf{k})\right) .
$$

To study the convergence of $d_{\rho}(s)$ we introduce the following functions

$$
\begin{gathered}
Z_{1}(s)=\exp \sum_{m=1}^{\infty} \frac{s^{m}}{m} \sum_{\sigma^{m} \mathbf{k}=\mathbf{k}} \frac{\exp \left(-\lambda_{u}^{m}(\mathbf{k})\right) \operatorname{tr} \rho^{m}(\mathbf{k})}{\left(\Delta^{m}(\mathbf{k}) \exp \lambda_{u}^{m}(\mathbf{k})-1\right)}, \\
Z_{2}(s)=\exp \sum_{m=1}^{\infty} \frac{s^{m}}{m} \sum_{\sigma^{m} \mathbf{k}=\mathbf{k}} \frac{\Delta^{m}(\mathbf{k}) \exp \left(\lambda_{s}^{m}(\mathbf{k})\right) \operatorname{tr} \rho^{m}(\mathbf{k})}{\left|\operatorname{det}\left(I-D f_{\mathbf{k}}^{m}\right)\right|},
\end{gathered}
$$




$$
Z(s, \rho)=\exp \sum_{m=1}^{\infty} \frac{s^{m}}{m} \sum_{\sigma^{m} \mathbf{k}=\mathbf{k}} \exp \left(-\lambda_{u}^{m}(\mathbf{k})\right) \operatorname{tr} \rho^{m}(\mathbf{k}) .
$$

We have $1 / d_{\rho}(s)=Z_{1}(s) Z_{2}(s) Z(s, \rho)$ and

$$
\begin{aligned}
\lim _{m \rightarrow \infty} & \frac{1}{m} \log \sum_{\sigma^{m} \mathbf{k}=\mathbf{k}} \frac{\exp \left(-\lambda_{u}^{m}(\mathbf{k})\right)}{\left|\Delta^{m}(\mathbf{k}) \exp \lambda_{u}^{m}(\mathbf{k})-1\right|} \\
= & \lim _{m \rightarrow \infty} \frac{1}{m} \log \sum_{\sigma^{m} \mathbf{k}=\mathbf{k}} \exp \left(-2 \lambda_{u}^{m}(\mathbf{k})\right)=P\left(-2 \lambda_{u}\right)<0, \\
\lim _{m \rightarrow \infty} & \frac{1}{m} \log \sum_{\sigma^{m} \mathbf{k}=\mathbf{k}} \frac{\exp \left(\lambda_{s}^{m}(\mathbf{k})\right)}{\left|\operatorname{det}\left(I-D f_{\mathbf{k}}^{m}\right)\right|} \\
= & \lim _{m \rightarrow \infty} \frac{1}{m} \log \sum_{\sigma^{m} \mathbf{k}=\mathbf{k}} \exp \left(\lambda_{s}^{m}(\mathbf{k})-\lambda_{u}^{m}(\mathbf{k})\right)=P\left(\lambda_{s}-\lambda_{u}\right)<0, \\
& \lim _{m \rightarrow \infty} \frac{1}{m} \log \sum_{\sigma^{m} \mathbf{k}=\mathbf{k}} \exp \left(-\lambda_{u}^{m}(\mathbf{k})\right)=P\left(-\lambda_{u}\right)=0 .
\end{aligned}
$$

Therefore $\log Z_{1}, \log Z_{2}$ have radius of convergence greater than $1 . \log Z(s, \rho)$ converges absolutely on the unit disc and so does $\log d_{\rho}$.

We now describe the techniques of Rugh [7], [8] and Fried [3]. First we introduce hyperbolic correspondences. We say that a correspondence $f \subset(I \times J) \times\left(I^{\prime} \times J^{\prime}\right)$ admits a cross map $c=(u, s): I^{\prime} \times J \rightarrow I \times J^{\prime}$ if

$$
f=\left\{\left(u\left(x^{\prime}, y\right), y, x^{\prime}, s\left(x^{\prime}, y\right): x^{\prime} \in I^{\prime}, y \in J\right\} .\right.
$$

If $s, u$ are restrictions of analytic contractions we say that $f$ is an analytic hyperbolic correspondence. When $\partial_{1} s=\partial_{2} u=0$, we say that $f$ is split. We will consider compositions of nearly split correspondences as follows.

Proposition 2. [3] Suppose that for $k=0,1,2, \ldots$ we have rectangles $I_{k} \times J_{k}$ and correspondences $f_{k} \subset\left(I_{k} \times J_{k}\right) \times\left(I_{k+1} \times J_{k+1}\right)$ which admit cross maps $c_{k}=\left(u_{k}, s_{k}\right)$. Put

$$
\alpha_{k}=\left\|\partial_{1} u_{k}\right\|, \beta_{k}=\left\|\partial_{2} u_{k}\right\|, \gamma_{k}=\left\|\partial_{1} s_{k}\right\|, \delta_{k}=\left\|\partial_{2} s_{k}\right\| .
$$

There is a positive function $\rho(\sigma)$ such that if $\alpha_{k}, \delta_{k} \leq \sigma<1$ and $\beta_{k}, \gamma_{k} \leq \rho(\sigma)$ for all $k$ then each composition $f_{m-1} \circ \cdots \circ f_{0}$ is a hyperbolic correspondence with cross map $c_{(0, \ldots, m-1)}=\left(u_{(0, \ldots, m-1)}, s_{(0, \ldots, m-1)}\right)$.

If the size of a Markov family $\left\{R_{k}\right\}$ with $R_{k} \subset D_{j}(k)$ is sufficiently small, each correspondence $f_{k l}$ extends to a hyperbolic correspondence between rectangles $I_{k} \times$ $J_{k}$ and $I_{l} \times J_{l}$ with cross maps $c_{k l}=\left(u_{k l}, s_{k l}\right)$ where $I_{k}, J_{k}$ are intervals in $T_{p_{k}} D_{j(k)} \cap$ $\left(E^{c} \oplus E^{u}\right)_{p_{k}}$ and $T_{p_{k}} D_{j(k)} \cap\left(E^{c} \oplus E^{s}\right)_{p_{k}}$ such that $I_{k} \times J_{k} \subset T_{p_{k}} D_{j(k)}$. To construct meromorphic continuation of the torsion function one considers sections of $\left|E^{u}\right| \otimes$ $\bigwedge^{i}\left(T M / E^{c}\right) \otimes E_{\rho} \mid D_{j}$. For $(k, l) \in A$ the transitions from $B_{l}$ to $B_{k}$ for $\bigwedge^{i}\left(T M / E^{c}\right)$ are given by

$$
\begin{gathered}
M_{k l}\left(1 ; x_{l}, y_{k}\right)=\frac{1}{\partial_{1} u_{k l}}\left(\begin{array}{cc}
1 & \partial_{1} s_{k l} \\
-\partial_{2} u_{k l} & \operatorname{det} D c_{k l}
\end{array}\right), \\
M_{k l}\left(0 ; x_{l}, y_{k}\right)=1, \quad M_{k l}\left(2 ; x_{l}, y_{k}\right)=\operatorname{det} M_{k l}\left(1 ; x_{l}, y_{k}\right)=\frac{\partial_{2} s_{k l}}{\partial_{1} u_{k l}} .
\end{gathered}
$$

Denote $\tau_{k l}\left(x_{l}, y_{k}\right)=t_{k l}\left(u_{k l}\left(x_{l}, y_{k}\right), y_{k}\right)$. One chooses discs $W_{k}, V_{k}$ in $\mathbb{C}$ containing $I_{k}, J_{k}$ and holomorphic extensions of $c_{k l}$ and $\tau_{k l}$ to $W_{l} \times V_{k}$. 
Denote by $\hat{\mathbb{C}}$ the Riemann sphere. Let $\mathcal{B}_{k}$ be the space of holomorphic functions on $\left(\hat{\mathbb{C}} \backslash \overline{W_{k}}\right) \times V_{k}$ vanishing on $\{\infty\} \times V_{k}$ that extend continuously to the boundary. Define $G_{k l}^{z}: W_{k}^{*} \times V_{k} \times W_{l} \times V_{l}^{*} \rightarrow \mathbb{C}$ where $W_{k}^{*}, V_{l}^{*}$ are neighborhoods of $\hat{\mathbb{C}} \backslash W_{k}$ and $\hat{\mathbb{C}} \backslash V_{l}$ by

$$
G_{k l}^{z}\left(w_{k}, v_{k}, w_{l}, v_{l}\right)=\frac{\partial_{1} u_{k l}\left(w_{l}, v_{k}\right) \exp \left(-z \tau_{k l}\left(w_{l}, v_{k}\right)\right)}{\left(w_{k}-u_{k l}\left(w_{l}, v_{k}\right)\right)\left(v_{l}-s_{k l}\left(w_{l}, v_{k}\right)\right)} .
$$

One defines transfer operators $\mathcal{L}_{j}(z) ; j=0,1,2$ on $\mathcal{U}_{0}=\mathcal{U}_{2}=\bigoplus_{k \in I} \mathcal{B}_{k} \otimes \mathbb{C}^{n}$ and $\mathcal{U}_{1}=\bigoplus_{k \in I} \mathcal{B}_{k} \otimes \mathbb{C}^{2} \otimes \mathbb{C}^{n}$ by

$$
\left(\mathcal{L}_{j}(z) \xi\right)_{k}=\sum_{l:(k, l) \in A} \Delta_{k l} L_{k l}(j ; z) \otimes \rho_{k l}^{t} \xi_{l}
$$

where

$$
L_{k l}(j ; z) \eta_{l}\left(w_{k}, v_{k}\right)=\int_{\partial W_{l}} \int_{\partial V_{l}} \frac{d w_{l} d v_{l}}{(2 \pi i)^{2}} G_{k l}^{z}\left(w_{k}, v_{k}, w_{l}, v_{l}\right) M_{k l}\left(j ; w_{l}, v_{k}\right) \eta_{l}\left(w_{l} . v_{l}\right)
$$

Remark 1. Since $c_{k l}=\left(u_{k l}, s_{k l}\right),(k, l) \in A$ is a contraction, transfer operators improve the region of analyticity.

We have power series expansions for the "kernels" $G_{k l}^{z} M_{k l}$, for example

$$
G_{k l}^{0}\left(w_{k}, v_{k}, w_{l}, v_{l}\right) M_{k l}\left(2 ; w_{l}, v_{k}\right)=\sum_{m_{1}, m_{2}=0}^{\infty} E_{m_{1} m_{2}}^{k}\left(w_{k}, v_{k}\right) g_{m_{1} m_{2}}^{l}\left(w_{l}, v_{l}\right)
$$

where $E_{m_{1} m_{2}}^{k}\left(w_{k}, v_{k}\right)=\left(w_{k}-a_{k}\right)^{-m_{1}-1}\left(v_{k}-b_{k}\right)^{m_{2}}$ with $a_{k}, b_{k}$ the centers of $W_{k}, V_{k}$ and

$$
g_{m_{1} m_{2}}^{l}\left(w_{l}, v_{l}\right)=\int_{\partial V_{l}} \frac{d y}{2 \pi i} \frac{\partial_{2} s_{k l}\left(w_{l}, y\right)\left(u_{k l}\left(w_{l}, y\right)-a_{k}\right)^{m_{1}}}{\left(v_{l}-s_{k l}\left(w_{l}, y\right)\right)\left(y-b_{k}\right)^{m_{2}+1}} .
$$

One sees [7] that $g_{m_{1} m_{2}}^{l}$ are holomorphic on a neighborhood of $\overline{W_{l}} \times \hat{\mathbb{C}} \backslash V_{l}$ and that there is $r<1$ such that $\left\|g_{m_{1} m_{2}}^{l}\right\|<C r^{m_{1}+m_{2}}$. Thus we have convergent expansions

$$
L_{k l}(2 ; 0) \eta_{l}=\sum_{m_{1}, m_{2}=0}^{\infty} E_{m_{1} m_{2}}^{k}\left\langle g_{m_{1} m_{2}}^{l}, \eta_{l}\right\rangle
$$

where

$$
\left\langle g_{m_{1} m_{2}}^{l}, \eta_{l}\right\rangle=\int_{\partial W_{l}} \int_{\partial V_{l}} \frac{d w_{l} d v_{l}}{(2 \pi i)^{2}} g_{m_{1} m_{2}}^{l}\left(w_{l}, v_{l}\right) \eta_{l}\left(w_{l}, v_{l}\right) .
$$

This implies that $\mathcal{L}_{2}(0)$ is a nuclear operator of order zero and therefore a trace class operator and we can form the Fredholm determinant

$$
\operatorname{det}\left(I-\mathcal{L}_{2}(0)\right)=\exp -\sum_{m=1}^{\infty} \frac{\operatorname{tr}\left(\mathcal{L}_{2}(0)^{m}\right)}{m}=\prod_{\lambda}(1-\lambda)
$$

where the product runs over the eigenvalues of $\mathcal{L}_{2}(0)$ counted with multiplicity.

If $\beta \in \mathcal{U}_{2}^{*}, \xi \in \mathcal{U}_{2}$ let $\beta \mid \mathcal{B}_{k} \otimes \mathbb{C}^{n}=\sum_{i} \beta_{k}^{i} \otimes e_{i}$ and $\xi_{k}=\sum_{j} \xi_{k}^{j} \otimes e_{j}$ then

$$
\left\langle\mathcal{L}_{2}(0)^{*} \beta, \xi\right\rangle=\sum_{(k, l) \in A} \sum_{m_{1}, m_{2}=0}^{\infty} \sum_{i, j=1}^{n} \Delta_{k l}\left\langle\beta_{k}^{i}, E_{m_{1} m_{2}}^{k}\right\rangle\left\langle g_{m_{1} m_{2}}^{l}, \xi_{l}^{j}\right\rangle\left\langle e_{i}, \rho_{k l}^{t} e_{j}\right\rangle .
$$


Thus

$$
\left(\mathcal{L}_{2}(0)^{*} \beta\right) \mid \mathcal{B}_{l} \otimes \mathbb{C}^{n}=\sum_{k:(k, l) \in A} \sum_{m_{1}, m_{2}=0}^{\infty} \sum_{i=1}^{n} \Delta_{k l}\left\langle\beta_{k}^{i}, E_{m_{1} m_{2}}^{k}\right\rangle g_{m_{1} m_{2}}^{l} \otimes \overline{\rho_{k l}} e_{i}
$$

is analytic on a neighborhood of $\overline{W_{l}} \times \hat{\mathbb{C}} \backslash V_{l}$.

Remark 2. Transfer operators have the functorial property.

This implies for $\mathbf{k}=\left(k_{0}, \ldots, k_{m}\right)$ with $\left(k_{i}, k_{i+1}\right) \in A$

$$
\begin{aligned}
& L_{\mathbf{k}}^{m}(j ; z) \eta_{k_{m}}(w, v):=L_{k_{0} k_{1}}(j ; z) \cdots L_{k_{m-1} k_{m}}(j ; z) \eta_{k_{m}}(w, v) \\
& \quad=\int_{\partial W_{k_{m}}} \int_{\partial V_{k_{m}}} \frac{d w^{\prime} d v^{\prime}}{(2 \pi i)^{2}} G_{\mathbf{k}}^{z}\left(w, v, w^{\prime}, v^{\prime}\right) M_{\mathbf{k}}\left(j ; w^{\prime}, v\right) \eta_{l}\left(w^{\prime}, v^{\prime}\right)
\end{aligned}
$$

where $M_{\mathbf{k}}$ is defined as $M_{k l}$ with $c_{k l}$ replaced by $c_{\mathbf{k}}=\left(u_{\mathbf{k}}, s_{\mathbf{k}}\right)$,

$$
G_{\mathbf{k}}^{z}\left(w, v, w^{\prime}, v^{\prime}\right)=\frac{\partial_{1} u_{\mathbf{k}}\left(w^{\prime}, v\right) \exp \left(-z \tau_{\mathbf{k}}\left(w^{\prime}, v\right)\right)}{\left(w-u_{\mathbf{k}}\left(w^{\prime}, v\right)\right)\left(v^{\prime}-s_{\mathbf{k}}\left(w^{\prime}, v\right)\right)},
$$

and $\tau_{\mathbf{k}}$ is a holomorphic extension of

$$
\tau_{\mathbf{k}}(x, y)=t_{k_{0} k_{1}}\left(u_{\mathbf{k}}(x, y), y\right)+\cdots+t_{k_{m-1} k_{m}} \circ f_{k_{m-2} k_{m-1}} \circ \cdots \circ f_{k_{0} k_{1}}\left(u_{\mathbf{k}}(x, y), y\right) .
$$

We have

$$
\operatorname{tr}\left(\mathcal{L}_{j}(z)^{m}\right)=\sum_{\sigma^{m} \mathbf{k}=\mathbf{k}} \Delta^{m}(\mathbf{k}) \operatorname{tr} \rho^{m}(\mathbf{k}) \operatorname{tr} L_{\mathbf{k}}^{m}(j ; z) .
$$

As proved by Rugh [7] and Fried [3] for $\sigma^{m} \mathbf{k}=\mathbf{k}$ we have

$$
\operatorname{tr} L_{\mathbf{k}}^{m}(j ; z)=-\frac{\exp \left(-z \tau^{m}(\mathbf{k})\right) \operatorname{tr}\left(\wedge^{j} D f_{\mathbf{k}}^{m}\right)}{\operatorname{det}\left(I-D f_{\mathbf{k}}^{m}\right)}
$$

Thus

$$
\begin{gathered}
\operatorname{tr}\left(\mathcal{L}_{0}(0)^{m}\right)=\sum_{\sigma^{m} \mathbf{k}=\mathbf{k}} \frac{\operatorname{tr} \rho^{m}(\mathbf{k})}{\left|\operatorname{det}\left(I-D f_{\mathbf{k}}^{m}\right)\right|}, \\
\operatorname{tr}\left(\mathcal{L}_{0}(z)^{m}\right)+\operatorname{tr}\left(\mathcal{L}_{1}(z)^{m}\right)+\operatorname{tr}\left(\mathcal{L}_{2}(z)^{m}\right)=-\sum_{\sigma^{m} \mathbf{k}=\mathbf{k}} \exp \left(-z \tau^{m}(\mathbf{k})\right) \Delta^{m}(\mathbf{k}) \operatorname{tr} \rho^{m}(\mathbf{k}) .
\end{gathered}
$$

Therefore the expression

$$
R_{\psi, \rho}(z)=\frac{\operatorname{det}\left(I-\mathcal{L}_{0}(z)\right) \operatorname{det}\left(I-\mathcal{L}_{2}(z)\right)}{\operatorname{det}\left(I-\mathcal{L}_{1}(z)\right)}
$$

provides a meromorphic continuation of $R_{\psi, \rho}(z)$. Similarly

$$
d_{\rho}(s)=\operatorname{det}\left(I-s \mathcal{L}_{0}(0)\right)=\prod_{\nu}(1-s \nu)
$$

where the product is taken over the eigenvalues of $\mathcal{L}_{0}(0)$, gives an analytic continuation of $d_{\rho}(s)$. 


\section{Special Value of the torsion Function}

In this section we prove Theorem 1 by using (3) and (5) to evaluate $R_{\phi, \rho}(0)$. Proposition 3 says that this value is the same we obtained in [9] under the assumption that the stable foliation was analytic. To use (5) we need the following lemmas.

Lemma 1. Let $\rho: \pi_{1}(M) \rightarrow U(n)$ be an acyclic representation. Then 1 is neither an eigenvalue of $\mathcal{L}_{0}(0)$ nor of $\mathcal{L}_{2}(0)$

Proof. Suppose 1 is an eigenvalue of $\mathcal{L}_{0}(0)$, then $s=1$ is a zero of $d_{\rho}(s)$ and so it is pole of $Z(s, \rho)$. We recall that in order to obtain a meromorphic continuation of $Z(s, \rho)$, one changes $\lambda_{u}$ to a Hölder cohomologous function $\Lambda_{u}$, depending only on future coordinates, and considers the Ruelle operators $\mathcal{N}$ and $\mathcal{N}_{\rho}$ defined on spaces of Hölder functions on $\Sigma_{A}^{+}$with values in $\mathbb{C}$ and $\mathbb{C}^{n}$ respectively by

$$
\begin{gathered}
(\mathcal{N} g)(\mathbf{k})=\sum_{\sigma \mathbf{l}=\mathbf{k}} \exp \left(-\Lambda_{u}(\mathbf{l})\right) g(\mathbf{l}) \\
\left(\mathcal{N}_{\rho} \eta\right)(\mathbf{k})=\sum_{\sigma \mathbf{l}=\mathbf{k}} \exp \left(-\Lambda_{u}(\mathbf{l})\right) \rho_{l_{0} k_{0}} \eta(\mathbf{l}) .
\end{gathered}
$$

Since $P\left(-\Lambda_{u}\right)=0$ we have that 1 is an eigenvalue of $\mathcal{N}$ with a positive eigenfunction $h$. According to [4] and [6], $Z(s, \rho)$ has a meromorphic continuation to a disc of radius $>1$ and its poles on that disc are the inverses $\lambda^{-1}$ of the eigenvalues of $\mathcal{N}_{\rho}$; therefore 1 is an eigenvalue of $\mathcal{N}_{\rho}$. Let $\xi$ be an eigenvector and put $\eta=\xi / h$, then

$$
\eta(\mathbf{k})=\sum_{\sigma \mathbf{l}=\mathbf{k}} \frac{h(\mathbf{k})}{h(\mathbf{l})} \exp \left(-\Lambda_{u}(\mathbf{l})\right) \rho_{l_{0} k_{0}} \eta(\mathbf{l})
$$

Let $K=\max \left\{|\eta(\mathbf{l})|: \mathbf{l} \in \Sigma_{A}\right\}$ and $\mathbf{r} \in \Sigma_{A}$ such that $|\eta(\mathbf{r})|=K$. If $|\eta(\mathbf{l})|<K$ for some $\mathbf{l}$ with $\sigma \mathbf{l}=\mathbf{r}$ then

$$
|\eta(\mathbf{r})| \leq \sum_{\sigma \mathbf{l}=\mathbf{r}} \frac{h(\mathbf{r})}{h(\mathbf{l})} \exp \left(-\Lambda_{u}(\mathbf{l})\right)|\eta(\mathbf{l})|<K .
$$

Therefore $|\eta(\mathbf{l})|=K$ if $\sigma^{m} \mathbf{l}=\mathbf{r}$. Since $\sigma$ is transitive $|\eta(\mathbf{k})|=K$ for any $\mathbf{k} \in \Sigma_{A}$. Thus the left-hand side of (6) is a convex combination of points in the sphere $S=\left\{x \in \mathbb{C}^{n}:|x|=K\right\}$ and the right-hand side is a point in $S$. We then have $\rho_{l_{0} l_{1}} \eta(\mathbf{l})=\eta \circ \sigma(\mathbf{l})$ for any $\mathbf{l} \in \Sigma_{A}$.

For $a \in I$ choose $\mathbf{l}$ periodic, say $\sigma^{m} \mathbf{l}=\mathbf{l}$, such that $l_{0}=a$ and let $\eta_{a}=\eta(\mathbf{l})$. If $\gamma$ is the corresponding periodic orbit of the flow we have

$$
\rho(\gamma) \eta_{a}=\eta_{a} .
$$

Given $\mathbf{k} \in \Sigma_{A}$ with $k_{0}=a$ define $\mathbf{k}^{j}=\left(k_{0}^{j}, k_{1}^{j}, \ldots\right)$ by $k_{s}^{j}=l_{s}$ if $s<j m$ and $k_{s+j m}^{j}=k_{s}$. Then $\mathbf{k}^{j}$ converges to $\mathbf{l}$ and

$$
\rho(\gamma)^{j} \eta\left(\mathbf{k}^{j}\right)=\eta(\mathbf{k})
$$

Since $\rho$ is unitary

$$
\lim _{j \rightarrow \infty}\left|\rho(\gamma)^{j} \eta\left(\mathbf{k}^{j}\right)-\eta_{a}\right|=\lim _{j \rightarrow \infty}\left|\rho(\gamma)^{j}\left(\eta\left(\mathbf{k}^{j}\right)-\eta_{a}\right)\right|=0
$$

therefore $\eta(\mathbf{k})=\eta_{a}$. Thus $\eta$ defines a nontrivial element of $H^{0}\left(M, E_{\rho}\right)$, which contradicts the acyclicity of $\rho$.

Now suppose that 1 is an eigenvalue of the compact operator $\mathcal{L}_{2}(0)$ then 1 is also an eigenvalue of $\mathcal{L}_{2}(0)^{*}$ and let $\beta$ be an eigenvector. According to Remark $1, \beta$ 
belongs to the space $\mathcal{U}^{\prime}=\bigoplus_{k \in I} \mathcal{B}_{k}^{\prime} \otimes \mathbb{C}^{n}$ where $\mathcal{B}_{k}^{\prime}$ is the space of analytic functions on $W_{k} \times \hat{\mathbb{C}} \backslash \overline{V_{k}}$ that extend continuously to the boundary. Restricted to $\mathcal{U}^{\prime}, \mathcal{L}_{2}(0)^{*}$ is given by

$$
\left(\mathcal{L}_{2}(0)^{*} \zeta\right)_{l}=\sum_{k:(k, l) \in A} \Delta_{k l} L_{k l}^{*} \otimes \overline{\rho_{k l}} \zeta_{k}
$$

where

$$
\begin{gathered}
L_{k l}^{*} \xi_{l}\left(w_{l}, v_{l}\right)=\int_{\partial W_{k}} \int_{\partial V_{k}} \frac{d w_{k} d v_{k}}{(2 \pi i)^{2}} H_{k l}\left(w_{k}, v_{k}, w_{l}, v_{l}\right) \xi_{l}\left(w_{k} . v_{k}\right), \\
H_{k l}\left(w_{k}, v_{k}, w_{l}, v_{l}\right)=\frac{\partial_{2} s_{k l}\left(w_{l}, v_{k}\right)}{\left(w_{k}-u_{k l}\left(w_{l}, v_{k}\right)\right)\left(v_{l}-s_{k l}\left(w_{l}, v_{k}\right)\right)} .
\end{gathered}
$$

We see that the restriction of $\mathcal{L}_{2}(0)^{*}$ to $\mathcal{U}^{\prime}$ is the operator $\mathcal{L}_{0}(0)$ for the reversed time flow and the contragradient representation $\rho^{*}=\rho$. As we have proved 1 is not an eigenvalue of this operator.

Lemma 2. The holomorphic exterior derivative d intertwines transfer operators at $z=0$ i.e. $\mathcal{L}_{j+1}(0) d=d \mathcal{L}_{j}(0)$ provided the left-hand side is defined.

Proof. From (4) we have

$$
\begin{aligned}
L_{k l}(0 ; 0) \eta_{l}\left(w_{k}, v_{k}\right) & =\int_{\partial W_{l}} \int_{\partial V_{l}} \frac{d w_{l} d v_{l}}{(2 \pi i)^{2}} \frac{\partial_{1} u_{k l}\left(w_{l}, v_{k}\right) \eta_{l}\left(w_{l}, v_{l}\right)}{\left(w_{k}-u_{k l}\left(w_{l}, v_{k}\right)\right)\left(v_{l}-s_{k l}\left(w_{l}, v_{k}\right)\right)}, \\
\left(\begin{array}{c}
\Xi_{k l}\left(w_{k}, v_{k}\right) \\
Z_{k l}\left(w_{k}, v_{k}\right)
\end{array}\right) & :=L_{k l}(1 ; 0)\left(\begin{array}{c}
\xi_{l} \\
\zeta_{l}
\end{array}\right)\left(w_{k}, v_{k}\right) \\
& =\int_{\partial W_{l}} \int_{\partial V_{l}} \frac{d w_{l} d v_{l}}{(2 \pi i)^{2}} \frac{\left(\begin{array}{c}
\xi_{l}+\partial_{1} s_{k l} \zeta_{l} \\
-\xi_{l} \partial_{2} u_{k l}+\operatorname{det} D c_{k l} \zeta_{l}
\end{array}\right)}{\left(w_{k}-u_{k l}\left(w_{l}, v_{k}\right)\right)\left(v_{l}-s_{k l}\left(w_{l}, v_{k}\right)\right)}, \\
L_{k l}(2 ; 0) \eta_{l}\left(w_{k}, v_{k}\right) & =\int_{\partial W_{l}} \int_{\partial V_{l}} \frac{d w_{l} d v_{l}}{(2 \pi i)^{2}} \frac{\partial_{2} s_{k l}\left(w_{l}, v_{k}\right) \eta_{l}\left(w_{l}, v_{l}\right)}{\left(w_{k}-u_{k l}\left(w_{l}, v_{k}\right)\right)\left(v_{l}-s_{k l}\left(w_{l}, v_{k}\right)\right)} .
\end{aligned}
$$

Integration by parts gives

(8)

$$
\begin{aligned}
\frac{\partial L_{k l}(0 ; 0) \eta_{l}}{\partial w_{k}} & =-\int_{\partial W_{l}} \int_{\partial V_{l}} \frac{d w_{l} d v_{l}}{(2 \pi i)^{2}} \frac{\partial_{1} u_{k l}\left(w_{l}, v_{k}\right) \eta_{l}\left(w_{l}, v_{l}\right)}{\left(w_{k}-u_{k l}\left(w_{l}, v_{k}\right)\right)^{2}\left(v_{l}-s_{k l}\left(w_{l}, v_{k}\right)\right)} \\
& =\int_{\partial V_{l}} \frac{d v_{l}}{(2 \pi i)^{2}} \int_{\partial W_{l}} \frac{d w_{l}}{w_{k}-u_{k l}\left(w_{l}, v_{k}\right)} \frac{\partial}{\partial w_{l}}\left(\frac{\eta_{l}\left(w_{l}, v_{l}\right)}{v_{l}-s_{k l}\left(w_{l}, v_{k}\right)}\right) \\
& =\int_{\partial W_{l}} \int_{\partial V_{l}} \frac{d w_{l} d v_{l}}{(2 \pi i)^{2}}\left[\frac{\partial_{1} \eta_{l}}{\left(w_{k}-u_{k l}\right)\left(v_{l}-s_{k l}\right)}+\frac{\eta_{l} \partial_{1} s_{k l}}{\left(w_{k}-u_{k l}\right)\left(v_{l}-s_{k l}\right)^{2}}\right] \\
& =\int_{\partial W_{l}} \int_{\partial V_{l}} \frac{d w_{l} d v_{l}}{(2 \pi i)^{2}} \frac{\partial_{1} \eta_{l}+\partial_{2} \eta_{l} \partial_{1} s_{k l}}{\left(w_{k}-u_{k l}\right)\left(v_{l}-s_{k l}\right)} .
\end{aligned}
$$

(9)

$$
\begin{aligned}
\frac{\partial L_{k l}(0 ; 0) \eta_{l}}{\partial v_{k}}= & \int_{\partial W_{l}} \int_{\partial V_{l}}\left[\partial_{2} \partial_{1} u_{k l}+\frac{\partial_{1} u_{k l} \partial_{2} u_{k l}}{w_{k}-u_{k l}}+\frac{\partial_{1} u_{k l} \partial_{2} s_{k l}}{v_{l}-s_{k l}}\right] \\
& \times \frac{\eta_{l}\left(w_{l}, v_{l}\right) d w_{l} d v_{l}}{(2 \pi i)^{2}\left(w_{k}-u_{k l}\right)\left(v_{l}-s_{k l}\right)} \\
= & \int_{\partial W_{l}} \int_{\partial V_{l}} \frac{d w_{l} d v_{l}\left[-\partial_{2} u_{k l} \partial_{1} \eta_{l}+\left(\partial_{1} u_{k l} \partial_{2} s_{k l}-\partial_{1} s_{k l} \partial_{2} u_{k l}\right) \partial_{2} \eta_{l}\right]}{(2 \pi i)^{2}\left(w_{k}-u_{k l}\right)\left(v_{l}-s_{k l}\right)} .
\end{aligned}
$$


(8) and (9) imply $d \mathcal{L}_{0}(0)=\mathcal{L}_{1}(0) d$.

$$
\begin{aligned}
\frac{\partial \Xi_{k l}}{\partial v_{k}}= & \int_{\partial W_{l}} \int_{\partial V_{l}} \frac{d w_{l} d v_{l}}{(2 \pi i)^{2}}\left[\frac{\partial_{2} \partial_{1} s_{k l} \zeta_{l}}{\left(w_{k}-u_{k l}\right)\left(v_{l}-s_{k l}\right)}\right. \\
& \left.+\left(\xi_{l}+\partial_{1} s_{k l} \zeta_{l}\right)\left(\frac{\partial_{2} u_{k l}}{\left(w_{k}-u_{k l}\right)^{2}\left(v_{l}-s_{k l}\right)}+\frac{\partial_{2} s_{k l}}{\left(w_{k}-u_{k l}\right)\left(v_{l}-s_{k l}\right)^{2}}\right)\right], \\
& \frac{\partial Z_{k l}}{\partial w_{k}}=\int_{\partial W_{l}} \int_{\partial V_{l}} \frac{d w_{l} d v_{l}}{(2 \pi i)^{2}} \frac{\xi_{l} \partial_{2} u_{k l}-\operatorname{det} D c_{k l} \zeta_{l}}{\left(w_{k}-u_{k l}\right)^{2}\left(v_{l}-s_{k l}\right)}
\end{aligned}
$$

As before

(10)

$$
\begin{aligned}
\frac{\partial \Xi_{k l}}{\partial v_{k}}-\frac{\partial Z_{k l}}{\partial w_{k}}= & \int_{\partial W_{l}} \int_{\partial V_{l}} \frac{d w_{l} d v_{l}}{(2 \pi i)^{2}}\left[\frac{\partial_{1} u_{k l} \partial_{2} s_{k l} \zeta_{l}}{\left(w_{k}-u_{k l}\right)^{2}\left(v_{l}-s_{k l}\right)}\right. \\
& \left.+\frac{\left(\xi_{l}+\partial_{1} s_{k l} \zeta_{l}\right) \partial_{2} s_{k l}}{\left(w_{k}-u_{k l}\right)\left(v_{l}-s_{k l}\right)^{2}}+\frac{\partial_{2} \partial_{1} s_{k l} \zeta_{l}}{\left(w_{k}-u_{k l}\right)\left(v_{l}-s_{k l}\right)}\right] \\
= & \int_{\partial W_{l}} \int_{\partial V_{l}} \frac{d w_{l} d v_{l}}{(2 \pi i)^{2}} \frac{\partial_{2} s_{k l}\left(w_{l}, v_{k}\right)\left(\partial_{2} \xi_{l}\left(w_{l}, v_{l}\right)-\partial_{1} \zeta_{l}\left(w_{l}, v_{l}\right)\right)}{\left(w_{k}-u_{k l}\left(w_{l}, v_{k}\right)\right)\left(v_{l}-s_{k l}\left(w_{l}, v_{k}\right)\right)}
\end{aligned}
$$

which implies $d \mathcal{L}_{1}(0)=\mathcal{L}_{2}(0) d$.

Propositions 1 and 3 (coming up) complete the proof of Theorem 1.

\section{Proposition 3.}

$$
R_{\phi, \rho}(0)=\frac{\operatorname{det}\left(I-\Delta\left(\gamma_{0}\right) \rho\left(\gamma_{0}\right)\right) \operatorname{det}\left(I-\rho\left(\gamma_{0}\right)\right)^{2}}{\operatorname{det}(I-H)} .
$$

Proof. Let $\mathcal{V}_{j}$ be the space of $\xi \in \mathcal{U}_{j}$ such that $\xi_{k}$ is analytic in a neighborhood of $\hat{\mathbb{C}} \backslash W_{k} \times \overline{V_{k}}$.

Let $\lambda \neq 0$ be an eigenvalue of $\mathcal{L}_{j}(0)$. If $\left(\mathcal{L}_{j}(0)-\lambda I\right) \xi=0$ then $\xi \in \mathcal{V}_{j}$. It follows by induction that $\left(\mathcal{L}_{j}(0)-\lambda I\right)^{k} \eta=0$ implies that $\eta \in \mathcal{V}_{j}$ and $\left(\mathcal{L}_{j}(0)-\lambda I\right)^{k} d \eta=0$.

If $\left(\mathcal{L}_{2}(0)-\lambda I\right)^{k} \eta=0$ for $\lambda \neq 0$, there is $\xi \in \mathcal{V}_{1}$ such that $\eta=d \xi$. Therefore

$$
\operatorname{det}\left(I-\mathcal{L}_{1}(0)\right)=\operatorname{det}\left(I-\mathcal{L}_{2}(0)\right) \operatorname{det}\left(I-\mathcal{L}_{1}(0) \mid \operatorname{ker} d \cap \mathcal{V}_{1}\right) .
$$

Let

$$
\omega_{k}=\frac{d w_{k}}{w_{k}-a_{k}} \quad \text { and } \quad \mathcal{H}=\left\{\xi \in \mathcal{U}_{1}: \xi_{k}=c_{k} \omega_{k}, c_{k} \in \mathbb{C}^{n}\right\} .
$$

It is straightforward to show that $\operatorname{ker} d \cap \mathcal{V}_{1}=d \mathcal{V}_{0} \oplus \mathcal{H}$ and for $\xi \in \mathcal{H}$ there is $\eta \in \mathcal{V}_{0}$ such that

$$
\left(\mathcal{L}_{1}(0) \xi\right)_{k}=\sum_{l:(k, l) \in A} \Delta_{k l} \rho_{k l}^{t} c_{l} \omega_{k}+d \eta_{k} .
$$

Since $\mathcal{L}_{1}(0) d=d \mathcal{L}_{0}(0)$ we have

$$
\operatorname{det}\left(I-\mathcal{L}_{1}(0) \mid \operatorname{ker} d \cap \mathcal{V}_{1}\right)=\operatorname{det}\left(I-\mathcal{L}_{0}(0)\right) \operatorname{det}(I-H)
$$

and so

$$
\operatorname{det}\left(I-\mathcal{L}_{1}(0)\right)=\operatorname{det}\left(I-\mathcal{L}_{2}(0)\right) \operatorname{det}\left(I-\mathcal{L}_{0}(0)\right) \operatorname{det}(I-H) .
$$

Since 1 is neither an eigenvalue of $\mathcal{L}_{0}(0)$ nor of $\mathcal{L}_{2}(0)$ and $I-H$ is nonsingular (Proposition 1) we have that 1 is not an eigenvalue of $\mathcal{L}_{1}(0)$. By (5) and (3) we finally obtain Proposition 3. 


\section{REFERENCES}

[1] Fried, D. Fuchsian Groups and Reidemeister Torsion. Contemp. Math. 53 (1986) 141-163. MR 88e: 58098

[2] Fried, D. Lefschetz formulae for Flows. Contemp. Math. 58 (1987) 19-69. MR 88k:58138

[3] Fried, D. Meromorphic Zeta Functions for Analytic Flows. Preprint 1993.

[4] Haydn, N.T.A. Meromorphic extension of the zeta function for Axiom A flows. Ergod. Th. and Dynam. Sys. 10 (1990) 347-360. MR 91g:58219

[5] Ratner, M. Markov decomposition for a Y-flow on a three-dimensional manifold. Math. Notes 6 (1968) 880-886.

[6] Ruelle, D. The Thermodynamic Formalism for Expanding Maps. Commun. Math. Phys. 125 (1989) 239-262. MR 91a:58149

[7] Rugh, H. The correlation spectrum for hyperbolic analytic maps. Nonlinearity 5 (1992) 1237-1263. MR 93i:58121

[8] Rugh, H. Generalized Fredholm Determinants and Selberg Zeta Functions for Axiom A Dynamical Systems. To appear in Ergod. Th. and Dynam. Sys.

[9] Sánchez-Morgado, H. Lefschetz formulae for Anosov flows on 3-manifolds. Ergod. Th. and Dynam. Sys. 13 (1993) 335-347.

Instituto de Matemáticas, Universidad Nacional Autónoma de México, Ciudad Universitaria C. P. 04510, MÉxico D. F., MÉxico

E-mail address: hector@gauss.matem.unam.mx 